\title{
DISPERSION CHARACTERISTICS OF LEAKY WAVES ON LOSSLESS AND LOSSY SLOTLINES
}

\author{
Ján Zehentner, Jan Macháč, Maurizio Migliozzi \\ Czech Technical University, Technická 2, 16627 Prague 6, Czech Republic
}

\begin{abstract}
This paper deals with the dependence of the type of solution of the determinantal slotline dispersion equation on the location of the poles of its matrix elements. A number of possible solutions above the cut-off frequencies of particular surface waves is determined. Complete dispersion characteristics of the 1 st and 2 nd leaky wave on the lossless and lossy slotline as well as the main characteristics of the 1 st up to the 4 th leaky wave on the lossless slotline are presented.
\end{abstract}

\section{INTRODUCTION}

The explanation of bound and leaky wave propagation on a slotline presented in [1] is convincing from the mathematical point of view. However, interpretation of the field behaviour on a lossless slotline is polemical from the physical standpoint, as [2] points out. Therefore, the question may be raised whether and how the bound wave converts into the leaky wave. Basic knowledge about this topic has been published in [3]. Individual authors have made additional contributions to the basic concept of the leaky waves. We have identified the 2 nd leaky wave on the lossless slotline [4].

In this paper complete real and complex solutions of the slotline dispersion equation for the 2nd leaky wave are presented. The fluent transition from the real to the complex improper solution of this equation is explained. The total number of these solutions after crossing the cutoff frequencies of the $\mathrm{TM}_{0}, \mathrm{TE}_{1}, \mathrm{TM}_{2}$, and $\mathrm{TE}_{3}$ surface waves is determined. The main dispersion characteristics of the 3rd and 4th leaky wave, newly identified on the slotline, are reported. The propagation constant for the 1 st and 2 nd leaky wave on the lossy slotline is specified.

\section{PROPERTIES AND BEHAVIOUR OF THE 2ND LEAKY WAVE SOLUTION}

We analysed the open slotline with the cross-section shown in Fig. 1 by the method of moments modified as in the Galerkin testing procedure in the spectral domain with successive

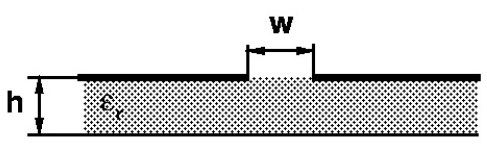

Fig. 1 Cross-section of a slotline.

complex root searching. First let us recall the normalized phase constant $\beta / k_{0}$ and the normalized leakage constant $\alpha / \mathrm{k}_{0}$ on the open lossless slotline, Fig. 2. Solution of the determinantal dispersion equation depends on the choice of the path of integration in the complex plane of the Fourier transform variable $\xi$ when its matrix elements are computed. All solutions belonging to the 2 nd leaky wave split off from the same type solution belonging to the 1st leaky wave at $f_{1}$, the cut-off frequency of the $T E_{1}$ surface wave, since the residue referred to leakage into the $T E_{1}$ surface wave is zero at $f_{1}$ This assertion holds for both the real solution and the complex solution. Owing to lack of space 
we do not show an example of the complex solution splitting which appears when the line has a wide slotwidth, e.g. for $w / h \geq 0.8$.

The complex improper solution of the $1 \mathrm{st}$ leaky wave begins at the real improper solution inside the spectral gap $f_{4}-f_{5}$. The real improper solution of the 2 nd leaky wave $\left(\beta / k_{0}<2\right.$ in Fig. 2$)$ breaks off from the 1 st leaky wave solution at
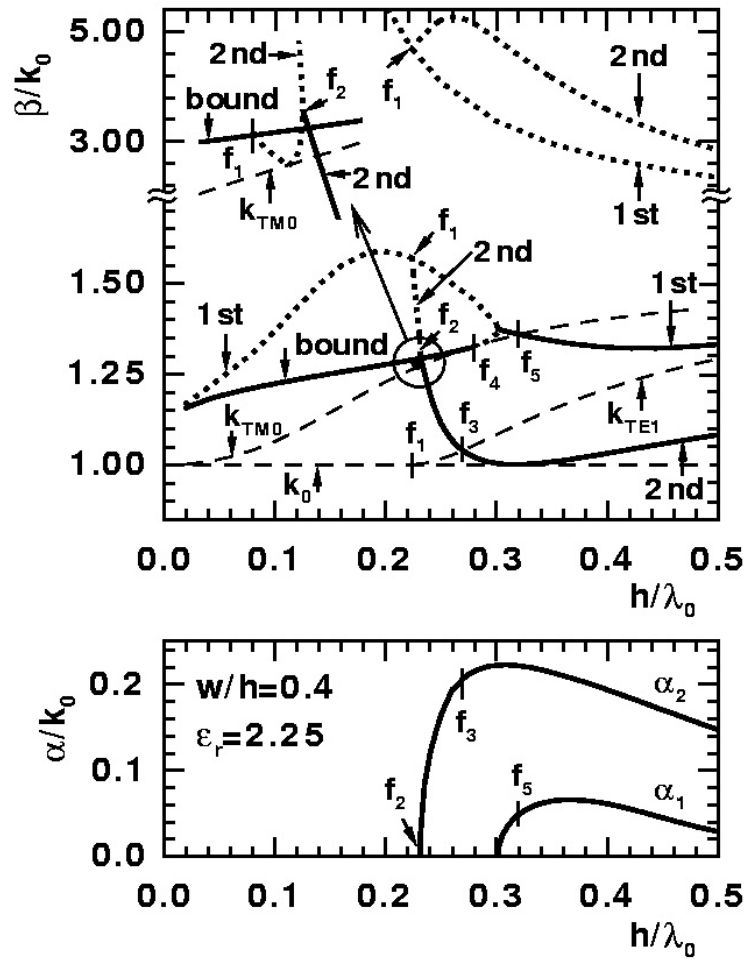

1 st and 2 nd improper complex 1 st and 2 nd improper real

Fig. 2 The normalized phase $\beta / \mathrm{k}_{0}$ and leaky $\alpha / \mathrm{k}_{0}$ constants for the slotline with $\mathrm{w} / \mathrm{h}=0.4, \varepsilon_{\mathrm{r}}=2.25$ as a function of normalized frequency $\mathrm{h} / \lambda_{\mathrm{O}}$.

$f_{1}$, then goes down, crosses the bound wave solution, touches the $\mathrm{k}_{\mathrm{TM}}$ curve, turns back and ends on the bound wave solution again at $f_{1}$. Only the pole of the Green function associated with the $\mathrm{TE}_{1}$ surface wave provides a solution in the last length between $f_{1}$ and $k_{\mathrm{TM} 0}$, while for all other 2nd leaky wave solutions poles related with the $\mathrm{TM}_{0}$ and $\mathrm{TE}_{1}$ surface wave were necessary.

After crossing the cut-off frequency $f_{1}$ the 2nd leaky wave solutions split off from each corresponding 1st leaky wave solution. The number of solutions above the cut-off frequency is twice the number of solutions below this cutoff frequency. This predicament can also be extended to the dispersion characteristics of higher order leaky waves.

To understand the change of the real into the complex improper solution, it is necessary to observe the migration of poles of integrals of the matrix elements of the determinantal dispersion equation. Dispersion characteristics of the 1 st

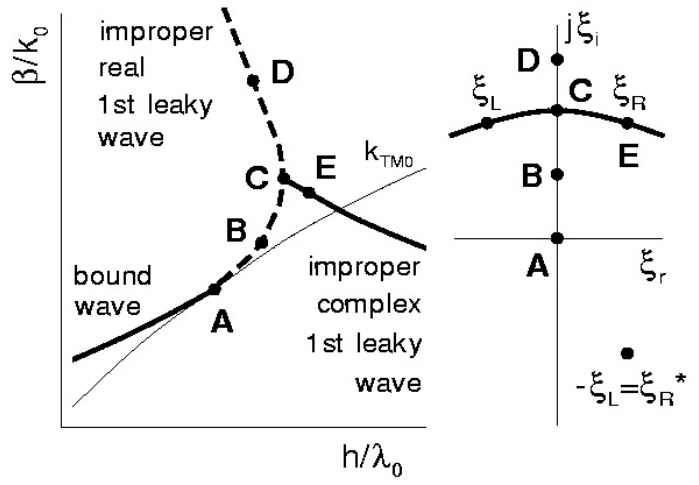

Fig. 3 A sketch of the dispersion characteristics of a lossless slotline inside and around the spectral gap and the corresponding migration of poles in the complex plane $\xi$ for the 1 st leaky wave.

leaky wave are sketched in Fig. 3. The bound wave solution results from calculations not including the residue in the $\mathrm{TM}_{0}$ pole. At the frequency corresponding to point $\mathrm{A}$, the $\mathrm{TM}_{0}$ pole is located in the origin of the complex plane $\xi$. With growing frequency, point B approaches point $\mathrm{C}$ and $\mathrm{TM}_{0}$ pole goes away from the origin along the imaginary axis. Coming from point $\mathrm{D}$ near to point $\mathrm{C}$ the pole sinks to the origin. At point $\mathrm{C}$ the two poles moving against one another collapse. After addition of a nonzero leakage constant $\alpha$ to $\beta$ at point $C$, the pole coming from the bottom/top to point $\mathrm{C}$ steps into the 1st/2nd quadrant. Now the poles are complex and $\xi_{\mathrm{R}}$ in the 1 st quadrant provides the improper solution $\gamma=\beta-j \alpha$ which is nonphysical inside the 
spectral gap. Since we are integrating an even function, it is sufficient to integrate in the interval $(0, \infty)$ instead of $(-\infty, \infty)$. To each pole there is also the pole located mirroredly to the origin. When the residue at the pole $\xi_{\mathrm{P}}{ }^{*}$, which is identical with the mirrored pole $-\xi_{\mathrm{L}}$, is accounted for, the second, complex conjugate solution $\gamma^{*}=\beta+j \alpha$ is obtained. The phase constant $\beta$ is double degenerated.

The explanation just given also holds for the 2 nd leaky wave when of course a couple of poles $\mathrm{TM}_{0}$ and $\mathrm{TE}_{1}$ have to be observed. At the frequency when the improper real solution of the 2nd leaky wave touches curve $\mathrm{k}_{\mathrm{TM} 0}$, pole $\mathrm{TM}_{0}$ is at the origin while pole $\mathrm{TE}_{1}$ lies at the imaginary axis. Similar migration of poles with increasing frequency has been observed also for the 3rd leaky wave, reported in the next paragraph, in the vicinity of point $\mathrm{G}$ in Fig. 4, at which two real solutions convert into one complex solution. This behaviour is reciprocal with respect to the frequency at point $\mathrm{F}$ in Fig. 4, when one complex solution converts into two real solutions.

\section{RD AND 4TH LEAKY WAVE ON THE SLOTLINE}

Analogously to the 2nd leaky wave we denote the field taking away power simultaneously to the $\mathrm{TM}_{0}, \mathrm{TE}_{1}, \mathrm{TM}_{2}$ surface wave as the 3rd leaky wave. We have identified its dispersion characteristics, some of which are shown in Fig. 4. Its excitation is possible on higher-permittivity substrates and at higher frequencies. Propagation of the bound wave and the 2nd leaky wave in Fig. 4 is possible from 45.8 $\mathrm{GHz}$ to $59.5 \mathrm{GHz}$. From $66.1 \mathrm{GHz}$ to 155.9 $\mathrm{GHz}$ the 1st and the 2nd leaky wave can propagate. The 3rd leaky wave can propagate together with the 1st leaky wave at frequencies greater than $163.0 \mathrm{GHz}$. However, already at frequency $118.8 \mathrm{GHz}$ the 4th leaky wave taking away power into the $\mathrm{TM}_{0}, \mathrm{TE}_{1}, \mathrm{TM}_{2}, \mathrm{TE}_{3}$ can excite. It is seen that the influence of the $3 \mathrm{rd}$ resp. 4th leaky wave can be neglected in $\mathrm{mm}$ wave circuits.
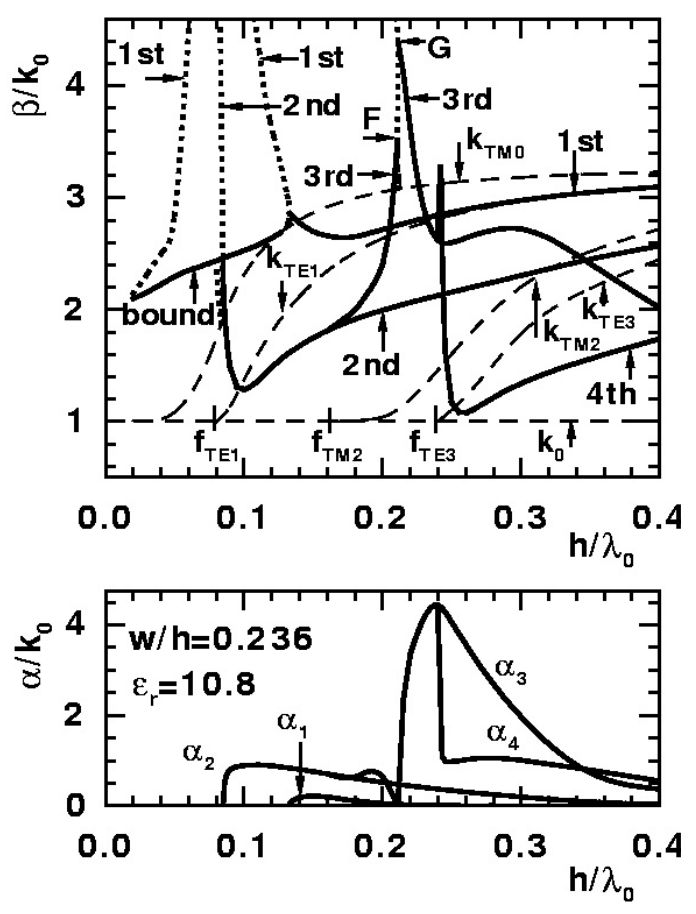

improper complex

improper real

Fig. 4 A plot similar to that in Fig. 2 now including solutions for the 1st, 2nd, 3rd and 4th leaky wave on the slotline with narrower slotwidth $\mathrm{w} / \mathrm{h}=0.236$ and higher substrate permittivity $\varepsilon_{\mathrm{r}}=10.8$.

\section{ST AND 2ND LEAKY WAVE ON THE LOSSY SLOTLINE}

For lossy substrate $\mathrm{k}_{\mathrm{TM} 0}$, the poles of the Green function and the propagation constant of the bound wave are complex. The bound wave solution continues as the improper complex solution. It is nonphysical inside the spectral gap and becomes physical above it, Fig. 5. Substrate losses cancel degeneration of $\beta$. Consequently the upper branch of the previous improper real solution (point D in Fig. 3) does not meet the lower branch (as at point $\mathrm{C}$ in Fig. 3) and now provides a nonphysical improper complex solution for all frequencies. Great substrate losses cause the fluent continuation of the bound wave solution as the physical improper complex solution of the 1st leaky wave, and disappearance of the spectral gap. Solutions for the 2 nd leaky wave are only complex and each of them begins 

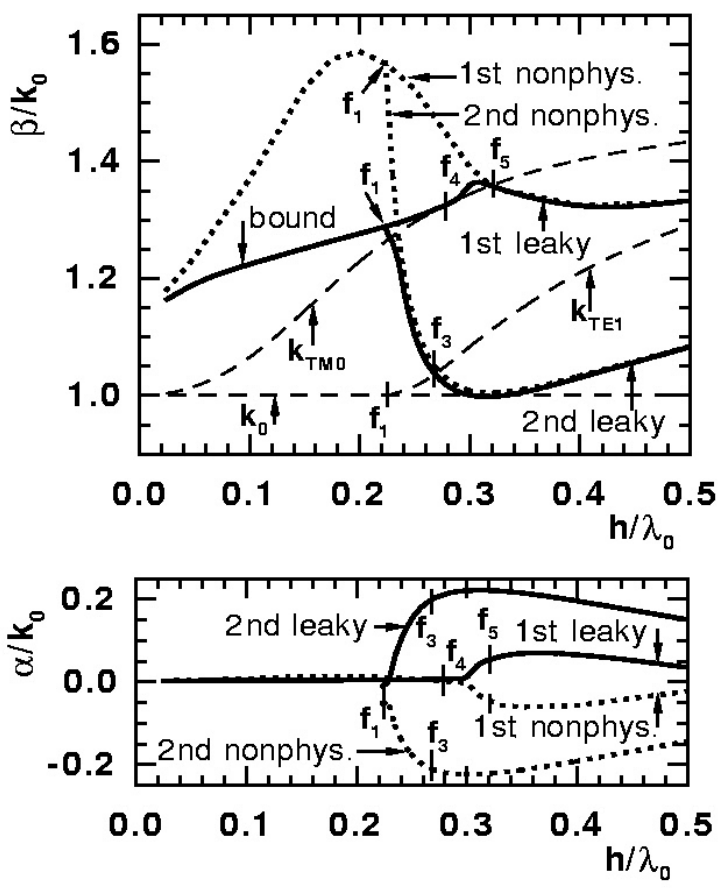

Fig. 5 A plot similar to that in Fig. 2 when $w / h=0.4$, $\varepsilon_{\mathrm{r}}=2.25$ but the substrate loss factor $\operatorname{tg} \delta=0.01$.

near to the corresponding 1st leaky wave solutions at the cut-off frequency of the $\mathrm{TE}_{1}$ surface wave, since the residue referred to leakage into the $\mathrm{TE}_{1}$ surface wave at this frequency is not zero. Only solutions beginning close to the bound wave solution are physical for frequencies greater than $f_{3}$, Fig. 5 .

Field attenuation owing to leakage and due to substrate losses overlap when leaky waves propagate over the lossy slotline. When e.g. $\operatorname{tg} \delta=0.01$ the 1 st leaky wave is physical at all frequencies above the spectral gap as in Fig. 5. The leaky wave converts back into the bound wave at higher frequencies. However, the physical principles of wave processes remain the same but the total field appears from the outside as the bound wave in accordance with [3]. The greater the loss factor, the lower is the frequency at which this conversion sets in and the spectral gap disappears simultaneously. Unfortunately, we have found out that leakage and substrate attenuation constants cannot be separated mathematically from each other, using both physical and nonphysical solutions, since they are now not complex conjugated.

\section{CONCLUSIONS}

The study of migration of poles of matrix elements of the slotline dispersion equation provides a varied explanation of the transition from the improper real to the complex solution for both the 1st and higher order leaky waves. New 3rd and 4th leaky waves have been identified on the slotline. The appearance of the main dispersion characteristics of the 1 st up to the 4th leaky wave indicates when these waves may be of interest in practice. Earlier ideas about the leaky wave on the lossy microstrip line are here confirmed for the 1 st and 2 nd leaky wave on the slotline.

\section{ACKNOWLEDGMENTS}

We gratefully acknowledge the scholarship granted by the Faculty of Electrical Engineering of the Czech Technical University in Prague to M. Migliozzi supporting his participation in this research. The work was done on the SP-2 computer at the Joint Supercomputer Center of the Czech Technical University, University of Chemical Technology and IBM Prague.

\section{REFERENCES}

[1] H. Shigesawa, M. Tsuji, A. A. Oliner: Dominant mode power leakage from printed-circuit waveguides. Radio Science, vol. 26, 1991, 2 Mar.-Apr., pp. 559564.

[2] A. A. Oliner, D. R. Jackson: On spectral gaps at the transition between bound and leaky modes. Proceedings of the 1995 International Symposium on Electromagnetic Theory, URSI, St. Petersburg, Russia, May 1995, pp. 764-766.

[3] H. Shigesawa, M. Tsuji, A. A. Oliner: The nature of the spectral gaps between bound and leaky solutions when dielectric loss is presented in printedcircuit lines. Radio Science, vol. 28, 1993, 6 Nov.Dec., pp. 1235-1243.

[4] J. Zehentner, J. Macháč, M. Migliozzi: Upper cutoff frequency of the bound wave and new leaky wave on the slotline. 1997 IEEE MTT-S IMS Digest, Denver, CO, vol. 2, pp. 487-490. 